
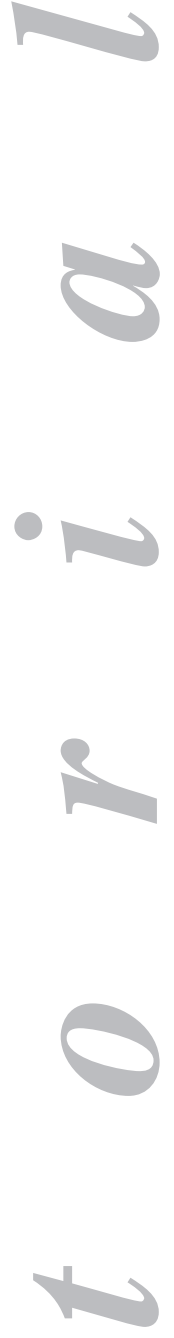

(
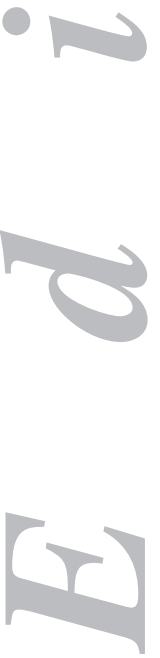

\section{Accuracy of colonoscopy in localizing colorectal cancer - A quality issue?}

Colorectal cancer (CRC) is a common, fatal disease. Around 33,000 patients are estimated to be newly diagnosed with colon cancer in Spain during 2012. While mortality from CRC has progressively decreased since 1990, it still is the second highest cause of cancer-related death. Early CRC is an indolent condition, with symptoms developing most commonly in advanced stages. Presentation typically varies according to tumor location. Overall, left-colon tumors manifest as rectorrhage and/or bowel rhythm changes. In contrast, right-colon tumors usually develop occult bleeding and secondary anemia. The vast majority of CRCs are endoluminal adenocarcinomas arising from the mucosa. Colonoscopy is the most accurate diagnostic test. It allows confirmation of tumor presence and its location, biopsy collection for histological study, and assessment of other synchronous lesions. On occasion, because of tumor growth, the malignancy blocks the intestinal lumen and prevents the colonoscope from advancing, thus rendering the examination incomplete. In such cases completing the examination using computed tomography (CT) colonography or magnetic resonance imaging (MRI) colonography is recommended. Once the diagnosis of CRC has been established, disease extension must be assessed both locally and remotely. Abdominal and pelvic CT, and some chest imaging technique are used for preoperative staging. The sensitivity of abdominal CT for distant metastatic disease identification is higher (75-87\%) than for node involvement detection (45-73\%) (1). The sensitivity of CT for primary tumor identification also decreases for smaller, flatter lesions. Regarding prognosis, numerous studies have tried to identify various histological, molecular, and clinical variables; however, pathological stage at diagnosis remains the best long-term prognostic marker for CRC to this day (2). Surgical resection is the only therapy with curative intent for localized CRC. Surgery may also be a potentially curative option in selected patients with metastatic disease confined to the liver and/or the lungs. The goal of colectomy with curative intent is complete tumor removal including its vascular pedicle and the lymphatic system draining the colonic segment involved. Although segmental resection may sufficient for primary tumor removal, wider resections are usually required for adequate lymphadenectomy. Resection margins, both distal and proximal, should at least measure $5 \mathrm{~cm}$ from the tumor, and allow an adequate resection of the intestinal segment involved together with its vascular supply and lymphatic drainage (3). Having these principles in mind, usual surgical procedures for colon cancer include:

- Right colectomy: for tumors located in the cecum and ascending colon, as well as some tumors at the hepatic flexure.

- Extended right colectomy: for tumors located in the hepatic flexure and proximal portion of the transverse colon.

- Transverse colectomy: (uncommon) for some tumors located in the middle of the transverse colon provided adequate resection margins and appropriate lymphadenectomy may be ensured. 
- Left colectomy: for tumors located in the distal portion of the transverse colon or the descending colon, and for selected patients with proximal tumors of the sigmoid colon.

- Sigmoid colectomy: for tumors located in the sigmoid colon sigmoid colectomy or segmental resection may be performed.

It is thus apparent that accurate tumor localization will determine surgery type with the purpose of achieving optimal therapy benefits while minimizing treatment-related morbidity.

In this setting two facts should be considered, which represent an improvement in the diagnosis, management, and prognosis of patients with CRC. On one hand the development of laparoscopic surgery. While laparoscopy-assisted colectomy was initially described in 1991, several studies were needed to demonstrate, over a decade later, that laparoscopic colectomy provides oncologic results that are comparable to those of open surgery (number of excised nodes, disease recurrence, etc.) as well as a number of added benefits, including reduced pain and hospital stay, as well as reduced ileus duration (4). Its widespread use in the future may therefore be anticipated. On the other hand, the implementation of screening programs for CRC in the population at average risk. These programs' goals include tumor detection during early stages, that is, when clinically silent and potentially curable. Preliminary results from the COLONPREV study, recently performed in a Spanish population to compare the usefulness of colonoscopy versus the fecal immunochemical test, confirm this hypothesis (5). Among tumors detected by these strategies, 96 and 83\%, respectively, corresponded to tumors in TNM classification stages I and II with no node involvement. Overall, these tumors are usually smaller than those diagnosed in symptomatic patients; some of them even correspond to malignant polyps with invasive carcinoma previously resected using polypectomy, for which therapy must be completed with surgery because of their not meeting good prognosis criteria. Therefore, to the extent these programs become widespread, a non-negligible percentage of patients undergoing surgery for CRC will possibly have small or even previously resected tumors. The coincidence in time of widespread laparoscopic surgery (with the limitation it entails for manual colonic examination) and the forseeable trend towards smaller tumors at diagnosis confirm the need to improve laparoscopic accuracy for CRC localization.

Thus, the study by Borda et al. published in this issue of the Journal is of particular interest (6). The authors perform a retrospective study of a significant number of tumors (237) diagnosed in patients with no history of prior colonic surgery. They review tumor location as estimated by colonoscopy and CT, and discuss consistency with actual sites as revealed by surgery, which they consider the gold standard. They also seek to determine which variables are associated with wrong endoscopic localizations. Although the retrospective, observational nature of the study might be thought of as a limitation regarding result generalization, other aspects in this paper demand attention. There is a tertiary, community hospital and endoscopies under scrutiny were carried out during the last five years, and so technical aspects (endoscopes, colon cleansing protocol) are presumably comparable to those found in most hospitals in our setting. The results reported regarding correct tumor localization using endoscopy very positively compare to those previously published in the literature, and are only second to results from two prior studies, one of which used additional means to estimate the endoscope's intracolonic position. Efforts to establish which variables are associated with incorrect localization are of paramount interest. Besides the tumor's obstructive nature, the other variable associated with incorrect localization was tumor location. Establishing which colon sites are more challenging to identify allows the endoscopist 
to remain more vigilant of such areas, attempting to define the most characteristic anatomic features, particularly when they are relevant in view of the surgical approach. Sites in the descending colon and the cecum are associated with a significantly increased risk for erroneous localization during endoscopy in the paper by Borda et al. Six tumors found in the cecum during surgery had been ascribed to the ascending colon by colonoscopy. In such a case surgical management remains unchanged since right hemicolectomy is indicated for both locations. However, errors in the localization of tumors of the descending colon may certainly be relevant regarding surgical management since, as reflected above, the surgical technique is different for tumors in the transverse colon and sigma. Interindividual variations in the anatomy of the descending colon may help explain such difficult localization, hence they must be understood in order to take them into account (7). In summary, the paper by Borda et al. may be said to describe a novel, relevant quality issue about colonoscopy that deserves further study in order to increase diagnostic accuracy for the anatomic localization of tumors in a consistent, reproducible manner.

M. Herráiz

Departament of Digestive Diseases. Unit of Prevention and High Risk Consultation of Digestive Tumors. Clínica Universidad de Navarra. Pamplona, Spain

\section{REFERENCES}

1. McAndrew MR, Saba AK. Efficacy of routine preoperative computed tomography scans in colon cancer. Am Surg 1999;65(3):205.

2. O'Connell JB, Maggard MA, Ko CY. Colon cancer survival rates with the new American Joint Committee on Cancer sixth edition staging. J Natl Cancer Inst 2004;96(19):1420.

3. Nelson H, Petrelli N, Carlin A, Couture J, Fleshman J, Guillem J, et al. Guidelines 2000 for colon and rectal cancer surgery. National Cancer Institute Expert Panel. J Natl Cancer Inst 2001;93(8):583.

4. Bonjer HJ, Hop WC, Nelson H, Sargent DJ, Lacy AM, Castells A, et al. Laparoscopically assisted vs. open colectomy for colon cancer: a meta-analysis. Transatlantic Laparoscopically Assisted vs. Open Colectomy Trials Study Group. Arch Surg 2007;142(3):298.

5. Quintero E, Castells A, Bujanda L, Cubiella J, Salas D, Lanas Á, et al. Colonoscopy versus fecal immunochemical testing in colorectal-cancer screening. N Engl J Med 2012;366(8):697-706.

6. Borda F, Jiménez FJ, Borda A, Urman J, Goñi S, Ostiz M, et al. Localización endoscópica del cáncer colorrectal: estudio de su precisión y posibles factores de error. Rev Esp Enferm Dig 2012;104(10):512-517.

7. Khashab MA, Pickhardt PJ, Kim DH, Rex DK. Colorectal anatomy in adults at computed tomography colonography: normal distribution and the effect of age, sex, and body mass index. Endoscopy 2009;41(8):674-8. 